\title{
Automated E-Commerce and Web Automation using Puppeteer
}

\author{
Arshdeep Singh Sachdeva ${ }^{1}$ Shreya Kapoor ${ }^{1}$ \\ Department of Information Technology, Maharaja Agrasen Institute of Technology, Delhi, India
}

\section{To Cite this Article}

Arshdeep Singh Sachdeva and Shreya Kapoor, "Automated E-Commerce and Web Automation using Puppeteer", International Journal for Modern Trends in Science and Technology, 6(12): 277-281, 2020.

\section{Article Info}

Received on 10-November-2020, Revised on 02-December-2020, Accepted on 06-December-2020, Published on 11-December-2020.

\section{ABSTRACT}

Automation replaces human work in repetitive, tedious tasks, and minimizes the quantity of errors. With the correct automation tools, it's possible to automate browser tasks, web testing, and online data extraction, to fill forms, scrape data, transfer data between applications, and generate reports. In this paper we will discuss about Automated E-Commerce website. The research project focuses on automating the task of placing an order of particular set of items from an online website. The idea is to save time and effort of the user by automating every task from signing in to adding products to the cart and filling up all the forms and placing order. The project also aims at providing the user a Real Time notification through mail for any Price Drops for a particular product that the user wishes to buy in future. Therefore, it also acts as a Price Monitoring System. There are various automation tools out of which we will discuss about Puppeteer.

KEYWORDS: Automation, Web Automation, Testing Tools, Web Testing, Puppeteer, Seleniu

\section{INTRODUCTION}

Web automation is that the concept of letting software robots perform actions, tasks, and processes that involves an online browser or web application. it's a process of automatically performing operations on an internet browser, so as to attain speed and efficiency levels that wouldn't be possible with human intervention.

The uses o browser automation are practically limitless, and if you are doing a big number of tasks throughout your work day, an automation tool may help reduce the time spent on these tasks while retrieving accurate and conclusive data. as an example, upon starting a browser you wish five different websites to be loaded to a particular point. rather than manually opening website, entering login credentials and navigating to a selected page, the automation tool can perform all of these tasks automatically.
Web Automation can even be used for testing purposes. Testing is a vital a part of every software development process on which companies devote considerable time and energy. With the appearance of internet revolution and therefore the colossal rise within the development of web applications additionally as their corresponding usage, it's becoming mandatory for quality testing of web applications.

Web Application Testing [1] is gaining importance given the most important stake of economic relevance within the contemporary society. The cost of fixing a bug is directly proportional to the time of its discovery. The longer the time it takes to unearth a bug, the costlier it becomes to fix it because the software would have been distributed or under use by the customers. 


\section{Structure of Paper}

The paper is organized as follows: In Section 1, the introduction of the paper is provided along with the structure, important terms, objectives and overall description. In Section 2 we have the complete information about automation, tools used in automation. Section 3 share information about Puppeteer, its advantages and disadvantages. Section 4 tells us about the methodology and the process description. Section 5 tells us about the future scope and concludes the paper with acknowledgement and references.

\section{Objectives}

Whether you're a software developer or just running several high-performing, application-rich websites, browser automation is swiftly becoming one amongst the foremost sought-after ways to test various site processes and codes. As Web-based technology evolves and becomes more dynamic, the necessity for dynamic testing solutions grows. While there are some ways to check the functionality of your website and applications, browser automation offers a way of performing such tasks without the requirement for manual manipulation. Ultimately, browser automation tools and techniques save developers hours in time as well as labor costs.

Therefore, the aim is to ease the task of a developer for testing purpose or to help the user perform daily life operations on the web easily by automating everything.

The idea is to save time and effort of the user by automating every task from signing in to adding products to the cart and filling up all the forms and placing order. The project also aims at providing the user a Real Time notification through mail for any Price Drops for a particular product that the user wishes to buy in future. Therefore, it also acts as a Price Monitoring System.

\section{AUTOMATION}

People use their browsers to access information and perform a good type of tasks. Browser automation [2] tools can automate your application program to perform repetitive and error-prone tasks, like filling out long HTML forms. Various skill levels will must be accommodated by the automation tool. A non-programmer might must simply record some test scripts, while programmers and advanced testers need more sophisticated scripts and libraries.
Web browser automation tools work by recording the series of steps that form up a selected transaction, and so play it back by injecting JavaScript into the target sites, and so tracking the providing the results. These web automation tools resemble macros, but are way more flexible and complicated.

Most things that you simply can do manually within the browser will be done using Puppeteer [3]

Here are some examples to induce you started:

- Generate screenshots and PDFs of pages.

- Crawl a SPA (Single-Page Application) and generate pre-rendered content (i.e. "SSR" (Server-Side Rendering)).

- Automate form submission, UI testing, keyboard input, etc.

- Create an up-to-date, automated testing environment. Run your tests directly within the latest version of Chrome using the most recent JavaScript and browser features.

- Capture a timeline trace of your site to assist diagnose performance issues.

- Test Chrome Extensions.

\section{Automation Tools [4]}

1. Puppeteer: Puppeteer is a Google product which allows you to manage Chrome with node scripts. you'll be able to automate headless instance of Chrome using this node library. It doesn't unlock anything new, but it abstracts many details you have got to cater to, without using it.

2. Selenium: Selenium also supports headless browser automation. because it doesn't have the GUI, user cannot see the screen of test execution. Selenium relies on Selenium IDE and Webdriver.

3. Protractor: Protractor could be a web automation framework to check angular applications. It supports locating elements which are specific to angular websites. Protractor also supports headless browser automation.

4. Katalon Studio: it's another automation tool which you'll use to check Web Application, API, Desktop applications and Mobile applications. Katalon studio also supports Headless browser testing. This tool is additionally built on top of Selenium.

\section{PUPPETEER}

We will be using Puppeteer [5] for our web automation because it provides the ability of headless chrome. Puppeteer is a Node library that 
gives a high-level API to manage Chrome or Chromium over the DevTools Protocol. It runs headless by default but may be configured to run full (non-headless) Chrome or Chromium.

Headless Chrome [6] is essentially a Chrome browser without UI. A Headless browser is more helpful for the programmers as they'll write the automation script very easily with up-to-date rendering of the scripts. It may be used for network throttling, device emulation and code coverage.

\section{How Puppeteer Outweighs Selenium [7]}

Selenium may be a widely used web automation tool, which supports different languages. Parallel testing is additionally possible in Selenium, and it's most useful after you automate an oversized number of browser instances, which are remotely distributed making it heavy. Also, object recognition in Selenium also becomes very difficult when the application is very big and changes are continuously occurring.

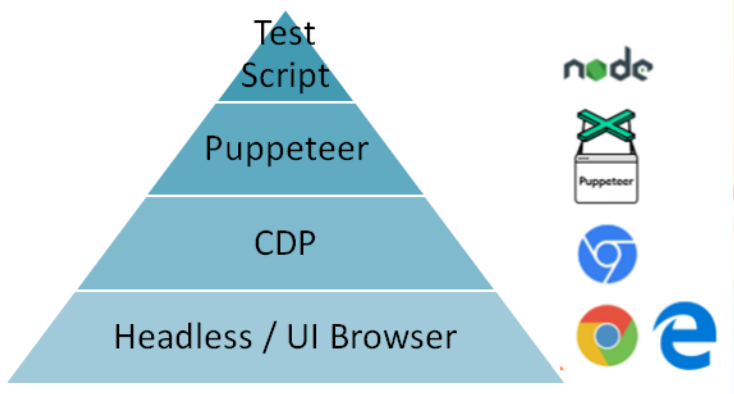

Advantages of Puppeteer over Selenium

- Puppeteer requires zero set-up effort and comes bundled with the Chromium version with which it works best, making it very easy to begin with. it's an event-driven architecture, which removes lots of potential synchronization issues.

- Puppeteer is a great tool for debugging: flip the "headless" bit to false, add "slowMo", and you'll see whatever is happening in the browser. Open Chrome DevTools to examine the test environment. It helps you to crawl a Single-Page Application and generate pre-rendered content (i.e. "SSR"Server-Side Rendering). It can easily automate form submissions, computer program testing, inputs from keyboard, etc.

- It can create an up-to-date, automated testing environment, run your tests within the latest version of Chrome using the most recent
JavaScript and every one new browser features. Puppeteer can capture a timeline trace of website to assist tracking performance bottlenecks and it's used for testing Chrome extensions.

- Puppeteer gives more power to control the Chrome browsers than Selenium Webdriver Puppeteer excludes the outbuilding on an external driver to run the tests. Despite this problem in Selenium, it can be reduced by using Boni Garcia's WebDriverManager dependency.

- You can test without loading the pictures within the application using Puppeteer tool which isn't possible in Selenium.

- By default, Puppeteer is about to execute in headless mode, but you can change/alter it for watching the execution board non-headless mode. It will be used for checking the proportion of CSS/JS files which are used for loading a page which isn't feasible in Selenium.

- Puppeteer also allows you to check the time taken to load the page but the identical feature isn't available in Selenium. It helps in testing new DevTools Protocol features and identifying bugs early. Executing the test in numerous devices using the emulators is feasible in Puppeteer but emulating a tool in Selenium is difficult.

\section{Puppeteer Limitations}

The complexity and automation context are changing with each passing day and hence, one tool may not be the answer for all. As every automation tool, Puppeteer has some limitations like, it supports only Chrome browser and Puppeteer Firefox is a work in progress. If the user base is more varied in its browser preferences, it should be wise to opt for other testing frameworks like Cypress.io, TestCafe or Selenium Web Driver.

\section{IV.METHODOLOGY}

The aim of the project is to provide hands-free experience to the users by automating each and every task that is done manually. The project focuses on taking input from the user, the list of products that the user wishes to order.

The idea is to take the input from user either from keyboard or by using Google's Speech API (to convert speech to text). With the integration of Google Speech API input is taken from the user in the form of speech input and then converted to 
text. All the products that are available at the website are added to the cart with the help of automated script and order is executed by filling up all the necessary details, forms etc. using the script.

Therefore, the project eases the task of placing an order on a website for a user by automating everything from speech input to adding desired products to the cart and filling up all the forms. This provides a completely hands-free experience to the users and they can just sit back and relax.

The project would also provide the user with real time notifications for a Price Drop of various products that the user wishes to buy. The project can also be used to compare prices of products from different sellers.

\section{Process Description}

- The user is required to input the data in from of either speech or text.

- The input is then processed by the automation script on a Headless Browser (such as Google Chromium).

- User will be automatically redirected to the home page of the online shopping website.

- User's login credentials saved in an encrypted form in the script would be retrieved and automatically typed on the login page of the website. User will be Logged In.

- The automated Script would search the different products by opening different tabs.

- All the products available on the website would be added to the cart as per the required quantity of the user.

- After adding all the products to the cart, the user will be proceeded to the payment page.

- Billing and Shipping details for the user will be automatically typed in.

- Other details such as contact and card details will also be filled automatically.

- Order would be successfully executed.

- Products that are either not available or out of stock would not be added to the cart and user will be notified about these products in the console.
We can keep the project running on our local system to get real time notification for Price Drops of various products. Further we can buy these products by using the above automated script.

\section{Limitations}

The whole process of automation is dependent on scraping/reading data from the websites on which we want to automate things. As these websites are Dynamic and their structure may change over time, so as a result our scripts would fail in that case.

We constantly need to keep the script updated so that it can find the required DOM elements from the webpage and perform automated tasks. The method of taking input in the form of speech can vary from person to person and can sometimes lead to incorrect results. The best possible results can thus be obtained by text input.or water floating temperatures. In recent years, it has been reported that heat causes changes in the mechanisms involved in regulating body fluids.

Pournot (2011) examined the short-term impacts of various immersions in recovery from intense activity. In this study, the effects of various immersion recovery techniques on the power and maximum power of athletes have been investigated, and the inflammatory response has been evaluated.

\section{FUTURE SCOPE AND CONCLUSION}

The whole browsing experience on the internet can be made hands-free with the help of speech input and using automation script. The user could do any task that is done manually by just using voice input from clicking buttons to opening websites, taking screenshots, generating PDF's etc. by just using their voice.

This could be a very useful and helpful feature for the people who could not type or have some disability, they could control everything on the web by just using their voice. Further the voice model could be trained to provide better experience to the users and provide more accurate results.

Automation is also used for web testing and can completely eliminate the role of test engineers by testing the websites with various test cases and check the durability and security of a website. 
Moreover, testing done with the help of automation will be more effective and accurate than manual testing.

\section{CONCLUSION}

The uses of browser automation are practically limitless, and if you are doing a large number of tasks throughout your work day, an automation tool may help reduce the time spent on these tasks while retrieving accurate and conclusive data.

With the advent of internet revolution and also the colossal rise within the development of web applications moreover as their corresponding usage, it's becoming mandatory for quality testing of web applications.

Web Application Testing is gaining importance given the main stake of economic relevance within the contemporary society. the value of fixing a bug is directly proportional to the time of its discovery. It provides speed and efficiency levels that wouldn't be possible with human intervention.

\section{Acknowledgement}

I am very thankful to Mrs. Sherya Kapoor who have contributed towards development of this template and have motivated and helped me in each and every step. I would also like to thank the pioneers of research in automation and testing of web applications who proposed several novel techniques and inspiring me to build a project using automation and further pursue a direction for effective testing of web applications.

\section{REFERENCES}

[1] Brian Marick. "When Should a Test Be Automated?". StickyMinds.com. Retrieved 2009-08-20.

[2] Browser Automation Tutorials, Green Technologyhttp://www.biodesignautomation.org/browser-automatio n-defined.html

[3] Puppeteer. (2020, June 24) Puppeteer Documentation. https://pptr.dev/

[4] Software Test Automation Abdul Rauf EMa,*, E.Madhusudhana Reddyb aResearch and Development Centre ,Bharathiyar University,Coimbatore-641014

[5] Google Web Developer Toolshttps://developers.google.com/web/tools/puppeteer.

[6] Headless Testing with Browsers https://docs.travis-ci.com/user/gui-and-headless-browse rs

[7] Aathira Shaji V S. (2020, Feb 17) "Puppeteer - Web Automation with Headless Chrome". RapidValue.https://www.rapidvaluesolutions.com/tech_b $\log$ /puppeteer-web-automation-with-headless-chrome/ 